\title{
On subparacompact and countably subparacompact spaces
}

\author{
M.K. Singal and Pushpa Jain
}

\begin{abstract}
A space is said to be subparacompact if every open covering of it has a $\sigma$-discrete closed refinement. Subparacompactness is equivalent to $F_{\sigma}$-screenability of McAuley and also to $\sigma$-paracompactness of Arhangel'skiT. Some properties of these spaces have been obtained in this note. Countably subparacompact spaces, which can be defined in an analogous manner, have also been studied.
\end{abstract}

In [12] MicAuley has introduced the concept of $F_{\sigma}$-screenability. He calls a space $X, F_{\sigma}$-screenable if every open covering of $X$ has a $\sigma$-discrete closed refinement. Obviously every regular paracompact space is $F_{\sigma}$-screenable. Bing [2] has given an example (Example H, [2]) to show that there exists a normal $F_{\sigma}$-screenable space which is not paracompact. However, as proved by McAuley [12], in a collectionwise normal space $F_{\sigma}$-screenability implies paracompactness. Again in [1], Arhangel'ski $Y$ introduced the notion of $\sigma$-paracompactness. According to him, a space $X$ is $\sigma$-paracompact if it has the property that 'for every open covering $u$ of $X$ there exists a sequence $\left\{u_{n}\right\}_{n=1}^{\infty}$ of open coverings of $X$ such that for each $x \in X$ there exists a positive integer $m(x)$ and a $U \in U$ such that $s t\left(x, u_{m(x)}\right) \subset U^{\prime}$, where $s t\left(x, u_{m(x)}\right)$ denotes the union of all those members of $u_{m(x)}$ which contain $x$. Burke and Stoltenberg [5] and 
Čoban [6] proved simultaneously, but independently, that $F_{\sigma}$-screenability implies $\sigma$-paracompactness. But, later on, Burke [3] proved that $F_{\sigma}$-screenability and $\sigma$-paracompactness are actually equivalent. He further proved that any of these properties of a space $X$ is equivalent to any of the following two properties of $X$ :

(1) every open covering of $X$ has a $\sigma$-locally finite closed refinement;

(2) every open covering of $X$ has a $\sigma$-closure preserving closed refinement.

A space characterized by any of the above four properties is called by Burke [3] a subparacompact space. Subparacompactness has also been studied recently by Hodel [9]. In the present note we present some further results on subparacompact spaces. In Section 1, some results concerning subparacompact subsets are obtained. Section 2 deals with some sum theorems. In Section 3 countably subparacompact spaces have been studied.

\section{Subsets and subparacompactness}

As with the other compactness properties, it can easily be verified that every closed subset of a subparacompact space is subparacompact. In fact, the following stronger result can be proved.

THEOREM 1.1. Every $F_{\sigma}$-subset (that is, a countable union of closed subsets) of a subparacompact space is subparacompact.

Proof. Let $X$ be a subparacompact space and let $A$ be an $F_{\sigma}$-subset of $X$. Let $U=\left\{U_{\alpha}: \alpha \in \Lambda\right\}$ be an open (in $A$ ) covering of $A$. Let $U_{\alpha}=A \cap U_{\alpha}^{\prime}$ for each $\alpha \in \Lambda$, where $U_{\alpha}^{\prime}$ is open in $X$. Since $A$ is an $F_{\sigma}$-subset, there exist closed subsets $A_{i}$ of $X$ such that $A=\bigcup_{i=1}^{\infty} A_{i}$. Now, for each $i$, let $\omega_{i}$ be an open covering of $X$ whose elements are $Y \sim A_{i}$ and all $U_{\alpha}^{\prime \prime s}$. Since $X$ is subparacompact, $W_{i}$ has a $\sigma$-locally finite closed refinement $v_{i}=\bigcup_{j=1}^{\infty} w_{i}^{j}$. Let $u_{i}^{j}$ be the collection of all 
those elements of $v_{i}^{j}$ which intersect $A_{i}$. Each $u_{i}^{j}$ is locally finite with respect to $X . \bigcup_{j=1}^{\infty} U_{i}^{j}$ is an open covering of $A_{i}$ such that every element of $u_{i}^{j}$ is contained in some $U_{\alpha}^{\prime}$. Let $u_{i}^{x^{j}}=\left\{B \cap A: B \in u_{i}^{j}\right\}$ and let $u^{*}=\bigcup_{i=1}^{\infty} \bigcup_{j=1}^{\infty} u_{i}^{* j}$. Then $u^{*}$ is a o-locally finite (in $A$ ) closed (in $A$ ) refinement of $U$. Hence $A$ is subparacompact.

DEFINITION 1.1. A subset $A$ of a topological space $X$ is said to be a generalized $F_{\sigma}$-subset if for each open subset $U$ of $X$ containing $A$ there exists an $F_{\sigma}$-subset $B$ of $X$ which is contained in $U$ and contains $A$.

THEOREM 1.2. Every generalized $F_{\sigma}$-subset of a subparacompact space is subparacompact:

Theorem 1.2 will easily follow from Theorem 1.1 and the following

THEOREM 1.3. Let $X$ be a topological space and let $A$ be a subset of $X$ such that every open subset of $X$ which contains $A$ contains $a$ subparacompact set which contains $A$. Then $A$ is subparacompact.

Proof. Let $U=\left\{U_{\alpha}: \alpha \in \Lambda\right\}$ be an open (in $A$ ) covering of $A$. For each $\alpha$, let $U_{\alpha}=A \cap V_{\alpha}$ where $V_{\alpha}$ is open in $X$. Then $A \subset \underset{\alpha \in \Lambda}{U} V_{\alpha}$. By the given condition there exists a subparacompact subset $B$ of $X$ such that $A^{\prime} \subset B \subset \underset{\alpha \in \Lambda}{\bigcup} V_{\alpha}$. Now $\left\{V_{\alpha} \cap B: \alpha \in \Lambda\right\}$ is an open covering of $B$. Thus it has a o-locally finite (in $B$ ) closed (in $B$ ) refinement $w=u_{i=1}^{\infty} w_{i}$. Let $w_{i}=\left\{w_{i j}: j \in \Delta_{i}\right\}$. If $w_{i j}=v_{i j} \cap B$, where $V_{i j}$ is closed in $X$, then $V_{i j} \cap A$ is closed in $A$. Also the family $V_{i}=\left(V_{i j} \cap A: j \in \Delta_{i}\right\}$ is locally finite in $A$ for each $i$, and $\infty$

$\underset{i=1}{U} V_{i}$ covers $A$. Hence $A$ is subparacompact. 
THEOREM 1.4. If every open subset of a subparacompact space $X$ is subparacompact, then every subset of $X$ is subparacompact.

Proof. Let $A$ be a subset of $X$ and let $U$ be an open (in $A$ ) covering of $A$. Then there is a collection $U^{*}$ of open subsets of $X$ such that $U=\left\{U^{*} \cap A: U^{*} \in U^{*}\right\}$. Let $G=U\left\{U^{*}: U^{*} \in U^{*}\right\}$. Then $G$ is an open subset of $X$ and $U^{*}$ is an open covering of $G$. Thus there is a sequence $\left\{u_{n}^{*}\right\}_{n=1}^{\infty}$ of open (in $G$ and hence in $X$ ) coverings of $G$ such that for each $x \in G$ there is a positive integer $m(x)$ and $U^{*} \in U^{*}$ such that $S t\left(x, u_{m(x)}^{*}\right) \subset U^{*}$. For each $n$, let $u_{n}=\left\{U^{*} \cap A: U^{*} \in U_{n}^{*}\right\}$. Then $\left\{u_{n}\right\}_{n=1}^{\infty}$ is a sequence of open (in $A$ ) coverings of $A$ satisfying the condition for subparacompactness of $A$.

\section{Sum theorems}

DEFINITION 2.1. (Katuta, [10]). A family $\left\{A_{\alpha}: \alpha \in \Lambda\right\}$ of subsets of $X$ is said to be order locally finite if there is a linear ordering ' $<$ ' of the index set $\Lambda$ such that for each $\alpha \in \Lambda$, the family $\left\{A_{\beta}: B<\alpha\right\}$ is locally finite at each point of $A_{\alpha}$.

Every o-locally finite family is order locally finite, but not conversely.

In [13], Singal and Arya have proved that for a topological property $P$ which is weakly hereditary (that is, a property which when possessed by a space is also possessed by every closed subset of it) and for which the locally finite sum theorem holds (that is, if $\left\{F_{\alpha}: \alpha \in \Lambda\right\}$ is a locally finite closed covering of $X$ such that each $F_{\alpha}$ has the property $P$, then $X$ has the property $P$ ), the following results are true:

THEOREM 2.1. Let $V$ be an order locally finite open covering of a space $X$ such that the closure of each member of $V$ possesses the property $P$. Then $X$ possesses $P$.

THEOREM 2.2. If $X$ is a regular space, if $V$ is an order zocally finite open covering of $X$ each member of which possesses the property $P$ and if the frontier of each member of $V$ is compact, then $X$ possesses 
the property $P$.

We shall prove that the locally finite sum theorem holds for subparacompactness.

THEOREM 2.3. If $\left\{F_{\alpha}: \alpha \in \Lambda\right\}$ is a locally finite closed covering of a space $X$ such that each $F_{\alpha}$ is subparacompact, then $X$ is subparacompact.

Proof. Let $U=\left\{U_{\beta}: \beta \in \Delta\right\}$ be an open covering of $X$. Then $\left\{U_{\beta} \cap E_{\alpha}: \beta \in \Delta\right\}$ is an open covering of $F_{\alpha}$ for each $\alpha$. Since $F_{\alpha}$ is subparacompact, therefore there exists a family $v^{\alpha}=\bigcup_{i=1}^{\infty} V_{i}^{\alpha}$ of closed subsets of $F_{\alpha}$ (and hence of $X$ ) such that each $V_{i}^{\alpha}$ is a discrete (in $F_{\alpha}$ and hence in $X$ ) family of subsets of $F_{\alpha}$ such that $V^{\alpha}$ is a covering of $F_{\alpha}$. For each $i$, let $\omega_{i}=\underset{\alpha \in \Lambda}{\cup} V_{i}^{\alpha}$ and let $\omega=\bigcup_{i=1}^{\infty} \omega_{i}$. Then $w$ is a closed covering of $X$ which is a refinement of $u$. Also, we shall show that each $w_{i}$ is locally finite. Let $x \in X$. Since $\left\{F_{\alpha}: \alpha \in \Lambda\right\}$ is locally finite, there exists an open set $M_{x}$ such that $M_{x} \cap F_{\alpha}=\emptyset$ for all except finitely many indices $\alpha$, say $\alpha_{1}, \alpha_{2}, \ldots, \alpha_{n}$. We can assume that $x \in F_{\alpha_{i}}$ for each $i=1,2, \ldots, n$. Since each one of the collections $v_{j}^{\alpha}, v_{j}^{\alpha}, \ldots, v_{j}^{\alpha}$ is discrete, therefore, for each $i=1,2, \ldots, n$ there exists an open set $W_{i}$ such that $x \in W_{i}$ and $W_{i}$ intersects at most one member of $v_{j}^{\alpha_{i}}$. Thus $x \in w_{1} \cap \ldots \cap w_{n} \cap M_{x}$ which is a neighbourhood of $x$ which intersects only finitely many members of $w_{i}$ and hence $w_{i}$ is locally finite. Thus $W$ is a $\sigma$-locally finite closed refinement of $u$ and hence $X$ is subparacompact.

COROLLARY 2.1. A disjoint topological sum of subparacompact spaces 
is subparacompact.

Since subparacompactness is weakly hereditary, it follows in view of Theorem 2.3 above that Theorems 2.1 and 2.2 are true for $P=$ subparacompact. Thus we have the following results.

THEOREM 2.4. If $V$ is an order locally finite open covering of $X$ such that the closure of each member of $V$ is subparacompact, then $X$ is subparacompact.

THEOREM 2.5. Let $X$ be a regular space and $V$ be an order locally finite open covering of $X$ such that each member of $V$ is subparacompact and the frontier of each member of $V$ is compact. Then $X$ is subparacompact.

Since every $\sigma$-locally finite family is order locally finite, we have the following important results as corollaries to Theorems 2.4 and 2.5 .

COROLLARY 2.2. Let $V$ be a o-locally finite open covering of a space $X$ such that the closure of each member of $V$ is subparacompact. Then $X$ is subparacompact.

COROLLARY 2.3. Let $X$ be a regular topological space and let $V$ be a o-locally finite open covering of $X$ such that each member of $V$ is subparacompact and the frontier of each member of $V$ is compact. Then $X$ is subparacompact.

DEFINITION 2.2. [Hodel, 8]. A subset $A$ of a space is said to be elementary if $i t$ is open and if there exists a sequence $\left\{A_{i}\right\}_{i=1}^{\infty}$ of open subsets of $X$ such that $A \subset \bigcup_{i=1}^{\infty} A_{i}$ and $\bar{A}_{i} \subset A$ for all $i$. A covering of $X$ consisting of elementary sets is said to be an elementary covering.

As a result of Theorem 2.3 and the weak hereditary character of subparacompactness, we have the following;

THEOREM 2.6. If $V$ is a o-locally finite elementary covering of $X$ such that each element of $V$ is subparacompact, then $X$ is subparacompact.

THEOREM 2.7. Let $V$ be a locally finite open covering of a regular space $X$ such that each member of $V$ is subparacompact and the frontier 
of each member of $V$ is Lindelöf. Then $X$ is subparacompact.

Proof. Let $U=\left\{V_{\alpha}: \alpha \in \Lambda\right\}$ be the given locally finite open covering of $X$. For each $\alpha \in \Lambda, \operatorname{FrV}_{\alpha}$ is Lindelöf. Therefore there exists a countable subfamily $\left\{V_{\alpha_{i}}: i=1,2, \ldots\right\}$ of $V$ which covers $\operatorname{Fr} V_{\alpha}$. Let $E_{1}=\operatorname{Fr} V_{\alpha} \sim \underset{i=2}{U} V_{\alpha_{i}}$, so that $F_{1}$ is a closed subset of $\operatorname{Fr} V_{\alpha}$ and hence of $X$ such that $F_{1} \subset V_{\alpha_{1}}$. We shall prove that there exists an open set $U_{1}$ such that $F_{1} \subset U_{1} \subset \bar{U}_{1} \subset v_{\alpha_{1}}$. Since $X$ is regular, for each $x \in F_{1}$ there is an open set $U_{x}$ such that $x \in U_{x} \subset \bar{U}_{x} \subset V_{\alpha_{1}}$. Since $F_{1}$ is Lindelöf, the open covering $\left\{U_{x}: x \in F_{1}\right\}$ of $F_{1}$ has a countable subcovering, say $U_{1}^{\prime}, U_{2}^{\prime}, \ldots$, such that for each $n \in N$, $\bar{U}_{n}^{\prime} \cap\left(x \sim V_{\alpha_{1}}\right)=\emptyset$. Now $X \sim v_{\alpha_{1}}=\left(X \sim \bar{V}_{\alpha_{1}}\right) \cup\left(\operatorname{FrV} \alpha_{\alpha_{1}}\right)$ and $\operatorname{Fr} V_{\alpha_{1}} \cap F_{1}=\varphi$, since $V_{\alpha_{1}}$ is open and $E_{1} \subset V_{\alpha_{1}}$. Let $y \in X \sim V_{\alpha_{1}}$. If $y \in \operatorname{Fr} V_{\alpha_{1}}$ then again by regularity of $x$ there exists an open set $V_{y}$ such that $y \in V_{y} \subset \bar{V}_{y} \subset X \sim F_{1}$. If $y \in X \sim \bar{V}_{\alpha_{1}}$, then $x \sim \bar{V}_{\alpha_{1}}$ is an open set containing $y$ such that $\overline{X \sim \bar{V}_{\alpha_{1}}} \cap F_{1}=\emptyset$; for if $z \in F_{1}$ then $V_{\alpha_{1}}$ is an open set containing $z$ such that $v_{\alpha_{1}} \cap\left(X \sim \bar{V}_{\alpha_{1}}\right)=\emptyset$, which implies $z \notin \overline{X \sim \bar{V}_{\alpha_{1}}}$. Since $\operatorname{Fr}_{\alpha_{1}}$ is Lindelö the covering $\left\{x \sim \bar{V}_{\alpha_{1}}, v_{y}: y \in \operatorname{Fr}_{\alpha_{1}}\right\}$ of $x \sim v_{\alpha_{1}}$ has a countable subcovering, say $V_{1}^{\prime}, V_{2}^{\prime}, \cdots$, which covers $x \sim V_{\alpha_{1}}$ and such that for each $n \in N$, $\bar{V}_{n}^{\prime} \cap F_{1}=\emptyset$. For each $n \in N$, let

$$
U_{n}^{n}=U_{n}^{\prime} \sim \cup\left\{\bar{V}_{p}^{\prime}: p \leq n\right\}
$$

and 


$$
V_{n}^{\prime \prime}=V_{n}^{\prime} \sim U\left\{\bar{U}_{p}^{\prime}: p \leq n\right\}
$$

Then $U_{n}^{\prime \prime} \cap V_{m}^{\prime \prime}=\psi$ for all $n, m \in N$. Let $U_{1}={\underset{n=1}{U}}_{n}^{\prime \prime}, V_{1}=\bigcup_{n=1}^{\infty} V_{n}^{\prime \prime}$ so that $E_{1} \subset U_{1}$ and $x \sim v_{\alpha_{1}} \subset v_{1}$. Hence $F_{1} \subset U_{1} \subset \bar{U}_{1} \subset x \sim v_{1} \subset v_{\alpha_{1}}$. $\bar{U}_{1}$, being a closed subset of $V_{\alpha_{1}}$, is subparacompact.

Now suppose that for eac $i=1,2, \ldots, n-1$, there exists an open set $U_{i}$ such that $E_{i} \subset U_{i} \subset \bar{U}_{i} \subset V_{\alpha_{i}}$ where

$$
F_{i}=\operatorname{Fr} V_{\alpha} \sim\left[\left(\int_{k=1}^{i-1} U_{k}\right) \cup\left(\begin{array}{cc}
u & V_{\alpha_{k}}
\end{array}\right)\right]
$$

and $\bar{U}_{i}$ is subparacompact. We shall now construct $U_{n}$ and $F_{n}$ as follows. Let $F_{n}=\operatorname{Fr} V_{\alpha} \sim\left[\left(\bigcup_{k=1}^{n-1} U_{k}\right) \cup\left(\begin{array}{cc}U_{k=n+1}^{\infty} & V_{\alpha_{k}}\end{array}\right)\right]$. Then $F_{n} \subset V_{\alpha_{n}}$, for if $x \in F_{n}$ then $x \in \bigcup_{k=1}^{\infty} V_{\alpha_{k}}, x \notin \bigcup_{k=1}^{n-1} U_{k}, x \notin \underset{k=n+1}{U} v_{k} . x \notin U_{n-1}$ implies that $\left.x \notin F_{n-1}=\operatorname{Fr} V_{\alpha} \sim\left[\int_{k=1}^{n-2} U_{k}\right) \cup\left(\begin{array}{cc}\infty & \\ u_{k=n} & v_{k}\end{array}\right)\right]$. Therefore $\infty$ $x \in \underset{k=n}{U} V_{\alpha_{k}}$ and thus $x \in V_{\alpha_{n}}$. As before, therefore, there exists an open set $U_{n}$ such that $F_{n} \subset U_{n} \subset \bar{U}_{n} \subset V_{\alpha_{n}}$ and $\bar{U}_{n}$ is subparacompact. The proof is complete by induction and we can define a family $u=\left\{U_{n}: n=1,2, \ldots\right\}$ of open sets satisfying

(a) $U$ is a covering of $\mathrm{FrV}_{\alpha}$;

(b) $\left\{\bar{U}_{n}: n=1,2, \ldots\right\}$ is locally finite. To prove (a), let $x \in \operatorname{Fr} V_{\alpha}$. Since $\left\{V_{\alpha_{i}}: i=1,2, \ldots\right\}$ is point finite, we can take the largest integer $i$ such that $x \in V_{\alpha_{i}}$. Then 
$x \in \bigcup_{j=1}^{i} V_{\alpha_{j}}$ and $x \bigcup_{j=i+1}^{\infty} V_{\alpha_{j}}$. In case $x \in \bigcup_{j=1}^{i-1} U_{j}$ we are done. Otherwise $x \in F_{i}$ and so $x \in U_{i} \cdot$ (b) is obvious in view of the fact that the family $\left\{\bar{U}_{n}: n=1,2, \ldots\right\}$ is a family of subsets of a locally finite family $\left\{V_{\alpha_{n}}: n=1,2, \ldots\right\}$.

$$
\text { Let } F_{0}=\bar{V}_{\alpha} \sim \bigcup_{k=1}^{\infty} U_{k} \text {; then }\left\{\bar{U}_{n}: n=1,2, \ldots\right\} \cup\left\{F_{0}\right\} \text { is a }
$$
locally finite closed covering of $\bar{V}_{\alpha}$ each nember of which is subparacompact. Hence $\bar{V}_{\alpha}$ is subparacompact. Thus $\left\{\bar{V}_{\alpha}: \alpha \in \Lambda\right\}$ is a locally finite closed covering of $X$ each member of which is subparacompact. Hence $X$ is subparacompact in view of Theorem 2.3.

DEFINITION 2.3. (Dowker [7]). A space $X$ is said to be totally normal if it is normal and if every open subset $G$ of $X$ is expressible as a union of a locally finite (in $G$ ) family of open $F_{\sigma}$-subsets of $X$.

It is proved in [7] that every subset of a totally normal space is totally normal.

THEOREM 2.8. Every subset of a totally normal subparacompact space is subparacompact.

Proof. In view of Theorem 1.4 we prove only that every open subset of a totally normal subparacompact space is subparacompact. Let $G$ be an open subset of $X$. Then $G=\underset{\alpha \in \Lambda}{U} G_{\alpha}$ where $\left\{G_{\alpha}: \alpha \in \Lambda\right\}$ is locally finite in $G$ and each $G_{\alpha}$ is an open $F_{\sigma}$-subset of $X$. Now $G$ is totally normal and hence normal. Therefore there exists a locally finite open refinement $\left\{H_{\alpha}: \alpha \in \Lambda\right\}$ of $\left\{G_{\alpha}: \alpha \in \Lambda\right\}$ such that $\vec{H}_{\alpha}^{G} \subset G_{\alpha}$ for each $\alpha \in \Lambda$, where $\vec{H}_{\alpha}^{G}$ denotes the closure of $H_{\alpha}$ in $G$. It can easily be seen that $\vec{H}_{\alpha}^{G}$ is an $F_{\sigma}$-subset of $X$. Therefore, by Theorem 2.3, it follows that $G$ is subparacompact. Hence the theorem. 


\section{Countably subparacompact spaces}

A space $X$ is said to be countably subparacompact if every countable open covering of $X$ has a $\sigma$-discrete closed refinement.

This definition is due to Hodel [9]. It is proved essentially in [11] that in a normal space countable subparacompactness, countable paracompactness and countable metacompactness are all equivalent. Also, in a screenable space (that is, a space in which every open covering has a o-mutually disjoint open refinement) countable subparacompactness is equivalent to subparacompactness ( $c f$. Hodel [9]). It can easily be verified that a space is countably subparacompact if and only if it satisfies the condition:

given a countable open covering $\left\{U_{n}: n=1,2, \ldots\right\}$ of $x$, there is a countable closed covering $\left\{F_{n j}: n=1,2, \ldots ; j=1,2, \ldots\right\}$ of $X$ with $F_{n j} \subset U_{n}$ for all $n$ and alz $j$.

In the present section we obtain some more characterizations of countable subparacompactness, and we find that if we add the word 'countable' with the open coverings in the equivalences for subparacompactness obtained by Burke [3] (mentioned in the introduction) we obtain the equivalences for countable subparacompactness [Theorem 3.1]. Throughout, $N$ will be used to denote the set of all natural numbers.

THEOREM 3.1. FOr a topological space $X$ the following are equivalent:-

(i) every countable open covering of $X$ has a o-discrete closed refinement;

(ii) every countable open covering of $X$ has a o-locally finite closed refinement;

(iii) every countable open covering of $X$ has a o-closure preserving closed refinement.

Before proving this theorem we prove the following lemma.

LEMMA 3.1. Let every countable open covering of $X$ have a closure preserving closed refinement. Then for every sequence $\{u(n)\}_{n=1}^{\infty}$ 
of countable open coverings of $x$, where $U(n)=\left\{U_{i}(n): i \in N\right\}$ and $U_{i}(n+1) \subset U_{i}(n)$ for all $i \in N$, there exists a sequence $\{V(n)\}_{n=1}^{\infty}$ of closed coverings of $X$ such that for each $n \in N, V(n)=\bigcup_{m=1}^{\infty} V_{m}(n)$ and the following are satisfied:-

(1) $v_{m}(n)=\left\{V_{i, m}(n): i \in N\right\}$ and $v_{m}(n)$ is closure preserving for each $m \in N$;

(2) $V_{i, m}(n) \subset U_{i}(n)$, for all $i$ and $m \in N$;

(3) $V_{i, m}(n) \subset V_{i, m+1}(n)$, for all $i$ and $m \in N$;

(4) $V_{i, m}(n+1) \subset V_{i, m}(n)$, for $a l 2 i$ and $m \in N$.

Proof of the lemma. Let $n \in N$. Let $P(n)=\bigcup_{m=1}^{\infty} P_{m}(n)$ be $a$ o-closure preserving closed refinement of $U(n)$. We now construct another $\sigma$-closure preserving closed refinement, $W(n)=\bigcup_{m=1}^{\infty} W_{m}(n)$ of $U(n)$ as follows: Let $w_{m}(n)=\bigcup_{i \leq m} P_{i}(n)$. Then $W(n)=\bigcup_{m=1}^{\infty} w_{m}(n)$ is a $\sigma$-closure preserving closed repinement of $u(n)$ such that $w_{m}(n) \subset \omega_{m+1}(n) \cdot$ Now, let $i, m, n \in N$. If $m<n$, let $V_{i, m}(n)=\varphi$. If $m \geq n$, define $V_{i, m}(n)=U\left\{W \in W_{m}(k): W \subset U_{i}(n), k \in N, n \leq k \leq m\right\}$.

Each $V_{i, m}(n)$, being a finite union of closed sets, is closed. Let $V_{m}(n)=\left\{V_{i, m}(n): i \in N\right\}$. Let $V(n)=\bigcup_{m=1}^{\infty} V_{m}(n)$. Then $\{V(n)\}_{n=1}^{\infty}$ is the required sequence of closed coverings.

Proof of Theorem 3.1. (i) $\Rightarrow$ (ii) and $(i i) \Rightarrow$ (iii) are obvious. Therefore we prove that $(i i i)=(i)$. Let $u=\left\{U_{i}: i \in N\right\}$ be a countable open covering of $X$. We shall construct a $\sigma$-discrete closed refinement 
of $u$. For each $n \in N$, let $U_{i}(1, n)=U_{i}$. Then $\{u(1, n)\}_{n=1}^{\infty}$, where $u(1, n)=\left\{U_{i}(1, n): i \in N\right\}$ is a sequence of countable open coverings of $X$. Thus, by the above lemma there exists a sequence $\{V(1, n)\}_{n=1}^{\infty}$ of countable closed coverings of $X$ such that for each $n \in N$, the following are satisfied:

$$
\begin{aligned}
& \text { (i) } v(1, n)=\bigcup_{m=1}^{\infty} v_{m}(1, n) ; \\
& \text { (ii) } v_{m}(1, n)=\left\{v_{i, m}(1, n): i \in N\right\} \text { and is closure preserving for } \\
& \text { each } m \in N ; \\
& \text { (iii) } V_{i, m}(1, n) \subset U_{i}(1, n) \text {, for all } i \text { and } m \in N ; \\
& \text { (iv) } V_{i, m}(1, n) \subset v_{i, m+1}(1, n) \text {, for all } i \text { and } m \in N ; \\
& \text { (v) } V_{i, m}(1, n+1) \subset V_{i, m}(1, n) \text {, for all } i \text { and } m \in N .
\end{aligned}
$$

By induction, therefore, it follows that for each $k \in N$, we can construct a sequence $\{V(k, n)\}_{n=1}^{\infty}$ of countable closed coverings of $X$ and a sequence of countable open coverings $\{U(k, n)\}_{n=1}^{\infty}$ such that for each $n \in N$, the following are satisfied:-

$$
\begin{aligned}
& \text { (1) } v(k, n)=\bigcup_{m=1}^{\infty} v_{m}(k, n) ; \\
& \text { (2) } v_{m}(k, n)=\left\{v_{i, m}(k, n): i \in N\right\} \text { and is closure preserving for } \\
& \text { each } m \in N ; \\
& \text { (3) } v_{i, m}(k, n) \subset U_{i}(k, n) \text {, for each } i \text { and } m \in N ; \\
& \text { (4) } v_{i, m}(k, n) \subset v_{i, m+1}(k, n) \text {, for each } i \text { and } m \in N ; \\
& \text { (5) } v_{i, m}(k, n+1) \subset v_{i, m}(k, n) \text {, for each } i \text { and } m \in N ; \\
& \text { (6) } U_{i}(k+1, n)=U_{i} \underset{j<i}{U} v_{j, n}(k, 1) \text {. }
\end{aligned}
$$

Each $u(k, n)$ is an open covering of $X$, for if $x \in X$ and $U_{i}$ is 
the first member of $u$ containing $x$, then $x \in U_{i}(k, n)$ for any $k, n \in N$. For $i, m, n, k \in N$, define

$$
W_{i}(k, m, n)=v_{i, m}(k, I) \cap v_{i, m}(k+1, n) \text {. }
$$

Then $w(k, m, n)=\left\{w_{i}(k, m, n): i \in N\right\}$ is a closure preserving collection of closed sets. Let $i, i^{\prime} \in N$ with $i^{\prime} \neq i$. Let $i^{\prime}<i$, then

$$
\begin{aligned}
w_{i}(k, m, n) & \subset v_{i, m}(k+1, n) \subset U_{i}(k+1, n) \\
& =U_{i} \sim u_{j<i} v_{j, n}(k, 1) \\
& \subset U_{i} \sim V_{i^{\prime}, n}(k, 1), \text { since } i^{\prime}<i \\
& \subset U_{i} \sim w_{i^{\prime}}(k, m, n) .
\end{aligned}
$$

Hence $w_{i},(k, m, n) \cap w_{i}(k, m, n)=\emptyset$ for all $i, i^{\prime} \in N$ with $i \neq i^{\prime}$. Thus $W_{i},(k, m, n)$, being a family of mutually disjoint closed sets, is discrete for each $k, m, n \in N$. Also $W_{i}(k, m, n) \subset U_{i}$. To prove that $W=\bigcup_{k=1}^{\infty} \bigcup_{m=1}^{\infty} \bigcup_{n=1}^{\infty} W(k, m, n)$ is a covering of $X$, let $x \in X$. Then there exists a $j \in N$ and $k \in N$ such that $x \in U_{j}(k, m)$ and $x \notin U_{i}(k, m)$ for $i<j$. Since $V(k, m)$ is a covering of $X$, there is an $n \in N$ such that $x \in V \in V_{n}(k, m)$. Then there is an $i \in N$ such that $x \in V_{i, n}(k, m)$ and $x \notin V_{i^{\prime}, n^{\prime}}\left(k^{\prime}, m^{\prime}\right)$ for $i^{\prime}<i$. We show that $x \in W_{i}(k, m, n)$. We notice that if $i^{\prime}<i$,

$$
\begin{aligned}
V_{i^{\prime}, m}(k+1, n) & \subset U_{i^{\prime}}(k+1, n) \\
& \subset U_{i^{\prime}} \sim V_{i, n}(k, 1),
\end{aligned}
$$

and so,

$$
V_{i^{\prime}, m}(k+1, n) \cap V_{i, n}(k, 1)=\emptyset \text {. }
$$

Thus 


$$
\begin{aligned}
x & \in V_{i, n}(k, m) \sim \underset{i^{\prime}<i}{U} V_{i^{\prime}, m}(k+1, n) \\
& \subset V_{i, n}(k, 1) \sim \underset{i^{\prime}<i}{\cup} V_{i^{\prime}, m}(k+1, n) \\
& =V_{i, n}(k, 1) \sim \underset{i^{\prime} \neq i}{U} V_{i^{\prime}, m}(k+1, n) \text {, in virtue of }(3) \text { and (6) } \\
& \subset V_{i, n}(k, 1) \cap V_{i, m}(k+1, n)=W_{i}(k, m, n) .
\end{aligned}
$$

Hence $\omega$ is a $\sigma$-discrete closed refinement of $U$; which proves (iii) $\Rightarrow$ (i).

THEOREM 3.2. A closed continuous image of a countably subparacompact space is countably subparacompact.

Proof. Let $f: X \rightarrow Y$ be a closed continuous mapping from a countably subparacompact space $X$ to a space $Y$. Let $U=\left\{U_{i}: i \in N\right\}$ be a countable open cover of $Y$. By continuity of $f$, $f^{-1}(u)=\left\{f^{-1}\left(U_{i}\right): i \in N\right\}$ is a countable open cover of $X$. Therefore there exists a closed refinement $P=U_{n=1}^{\infty} P_{n}$ of $f^{-1}(U)$ where each $P_{n}$ is a closure preserving collection of closed sets. Since $f$ is a closed mapping, $f\left(P_{n}\right)=\left\{f(P): P \in P_{n}\right\}$ is a closure preserving collection of closed sets in $Y$. Thus $f(P)$ is a $\sigma$-closure preserving refinement. Hence $Y$ is countably subparacompact.

THEOREM 3.3. If $f: X \rightarrow Y$ is a closed, continuous mapping from a regular space $X$ onto a countably subparacompact space $Y$ such that $f^{-1}(y)$ is compact for each $y \in Y$, then $X$ is countably subparacompact. Proof. Suppose $U=\left\{U_{i}: i \in N\right\}$ is an open cover of $X$. For each $y \in Y$, we can find a finite subcollection $U(y) \subset U$ such that $f^{-1}(y) \subset U(y)=U\{U: U \in U(y)\}$. Let $V(y)=Y \sim f(x \sim U(y))$. Then $V=\{V(y): y \in Y\}$ is an open cover of $Y$. Since $U$ is countable and $\{u(y): y \in Y\} \subset\{W: W$ is a finite subcollection of $U\}$ we see that $\{u(y): y \in Y\}$ is countable. Then $\{U(y): y \in Y\}$ and hence $V$ is countable. Thus, by countable subparacompactness of $Y, V$ has a 
$\sigma$-discrete closed refinement $V^{*}=\bigcup_{n=1}^{\infty} V_{n}^{*}$. Clearly $f^{-1}\left(V^{*}\right)=\left\{f^{-1}\left(V^{*}\right): V^{*} \in V^{*}\right\}$ is a $\sigma$-discrete refinement of $\{U(y): y \in Y\}$. Given $V^{*} \in V^{*}$, let $y\left(V^{*}\right)$ be a fixed element of $Y$ such that $f^{-1}\left(V^{*}\right) \subset U\left(y\left(V^{*}\right)\right)$. For each $n \in N$, let $u_{n}^{*}=\left\{f^{-1}\left(V^{*}\right) \cap U: V^{*} \in V_{n}^{*}, U \in U\left(y\left(V^{*}\right)\right)\right\}$. Since $f^{-1}\left(V_{n}^{*}\right)$ is a discrete collection in $X$, each $x \in X$ has a neighbourhood $N_{x}$ which intersects at most one element of $f^{-1}\left(V_{n}^{*}\right)$. Since each element of $f^{-1}\left(V_{n}^{*}\right)$ intersects only finitely many elements of $u_{n}^{*}$ and each element of $u_{n}^{*}$ is contained in some element of $f^{-1}\left(V_{n}^{*}\right)$ it follows that $N_{x}$ will intersect only finitely many elements of $u_{n}^{*}$. So $u_{n}^{*}$ is locally finite and

$u^{*}=\bigcup_{n=1}^{\infty} u_{n}^{*}$ is a $\sigma$-locally finite refinement of $u$. Since $x$ is regular, it follows that every countable open cover of $X$ has a $\sigma$-locally finite closed refinement. Hence $X$ is countably subparacompact.

REMARKS. It is easy to see that the results of Theorems $1.1,1.2$, $1.3,1.4,2.3,2.4,2.5,2.6,2.7,2.8$ and Corollaries $2.1,2.2,2.3$ in Sections 1 and 2 remain true if 'subparacompact' is replaced by 'countably subparacompact' .

\section{References}

[1] A.V. Arhangel'skiT, "Mappings and spaces" (Russian), Uspehi Mat. Nauk 21 (1966), no. 4 (130), 133-184; translated as Russian Math. Surveys 21 (1966), no. 4, 115-162.

[2] R.H. Bing, "Metrization of topological spaces", Canad. J. Math. 3 (1951), 175-186.

[3] Dennis K. Burke, "On subparacompact spaces", Proc. Amer. Math. Soc. $23(1969), 655-663$. 
[4] Dennis K. Burke, "Subparacompact spaces", Proc. Washington State Univ. Conf. General Topology, Washington, 1970, 39-49. (Edited and distributed by $\mathrm{Pi}$ Mu Epsilon, Pullman; Department of Mathematics, Washington State University, 1970.)

[5] D.K. Burke and R.A. Stoltenberg, "A note on p-spaces and Moore spaces", Pacific J. Math. 30 (1969), 601-608.

[6] M.M. Coban, " $\sigma$-paracompact spaces" (Russian), Vestnik Moskov. Univ. Ser. I Mat. Meh. 24 (1969), no. 1, 20-27.

[7] C.H. Dowker, "Inductive dimension of completely normal spaces", Quart. J. Math. Oxford (2) 4 (1953), 267-281.

[8] R.E. Hodel, "Sum theorems for topological spaces", Pacific J. Math. 30 (1969), 59-65.

[9] R.E. Hodel, "A note on subparacompact spaces", (to appear).

[10] Yûkiti Katuta, "A theorem on paracompactness of product spaces", Proc. Japan Acad. 43 (1967), 615-618.

[11] M.J. Mansfield, "On countably paracompact normal spaces", Canad. J. Math. 9 (1957), 443-449.

[12] Louis F. McAuley, "A note on complete collectionwise normality and paracompactness", Proc. Amer. Math. Soc. 9 (1958), 796-799.

[13] M.K. Singal and Shashi Prabha Arya, "Two sum theorems for topological spaces", Israel J. Math. 8 (1970), 155-158.

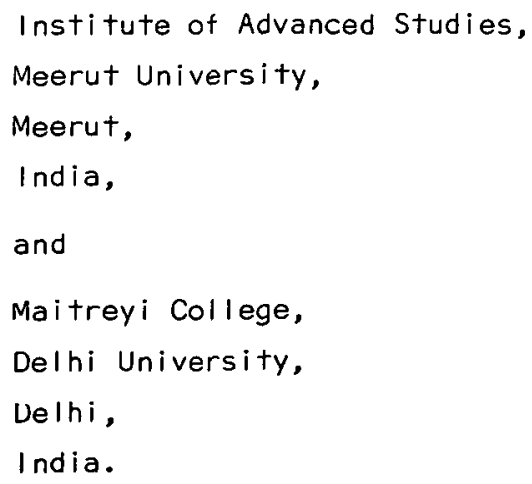

